\title{
Probing Plasmons in Three Dimensions within Random Morphology Nanostructures
}

Jordan Hachtel ${ }^{1,2}$, Anas Mouti ${ }^{2}$, Daniel Mayo ${ }^{3,4}$, Claire E. Marvinney ${ }^{3}$, Richard Mu$^{4}$, Stephen J. Pennycook $^{5}$, Andrew R. Lupini ${ }^{2}$, Matthew F. Chisholm ${ }^{2}$, Richard F. Haglund ${ }^{1,3}$, Sokrates T. Pantelides ${ }^{1,2,6}$

1. Vanderbilt University, Department of Physics and Astronomy, Nashville, TN USA

2. Oak Ridge National Laboratory, Materials Science and Technology Division, Oak Ridge, TN USA

3. Vanderbilt University, Interdisciplinary Materials Science Program, Nashville TN, USA

4. Fisk University, Department of Physics and Astronomy, Nashville, TN USA

5. National University of Singapore, Department of Materials Science and Engineering, Singapore

6. Vanderbilt University, Department of Electrical Engineering and Computer Science, Nashville, TN USA

Surface plasmon resonances (SPRs) are highly tunable and versatile in device applications, but also vulnerable to inhomogeneities and fabrication variances that can negatively influence the overall device performance. As a result, methods to study and analyze the plasmonic response in complex nanostructures with nanometer precision are needed. Scanning transmission electron microscopy (STEM) and STEM-based cathodoluminescence (CL) and electron energy loss spectroscopy (EELS) have proven to be valuable tools in nanoplasmonic analysis [1-3]. Both types of spectroscopy are useful in their own regard, but each suffers from its own set of limitations, restricting the possible analyses. However, the strengths and weaknesses of CL and EELS are complementary to one another, EELS is a measure of excitation, while CL is a measure of radiative decay. By comparing the two spectroscopies to one another, while keeping this distinction in mind, new information is available that is unavailable from the spectroscopies performed individually [4].

So far STEM-based investigations of SPRS have been limited to ordered structures and symmetric particles, or have relied on simulations to extract information from the data. Here we show that the combination of spatially-resolved STEM-CL and STEM-EELS analysis can be used to probe the plasmonic response of an irregularly shaped nanoparticle, randomly oriented relative to the electron beam, in three dimensions. The distinctive differences between the CL and EELS profiles allow us to observe and identify the dominant plasmon modes, and account both for the variations in intensity and for spatial distribution of the SPRs without the assistance of simulations.

Here we present a combined analysis for a single $\mathrm{Ag}$ nanoparticle on the surface of a $\mathrm{ZnO} / \mathrm{MgO}$ core/shell nanowire. The nanoparticle is selected for having high aspect ratios, thus generating nonoverlapping surface plasmon modes, as well as having a thick $\mathrm{MgO}$ barrier between the nanoparticle and nanowire to isolate the SPRs from charge-transfer interactions with the optically active $\mathrm{ZnO}$.

A high angle annular dark field (HAADF) image of the nanoparticle can be seen in Figure 1a, and next to it the CL and EELS maps of the three dominant longitudinal plasmon modes (Figure 1b-g). The differences between them are immediately apparent, but by combining the CL and EELS maps we can form an understanding of each mode. In Figure $1 \mathrm{~b}$ and 1c we see the CL and EELS profile of the $2.0 \mathrm{eV}$ peak. By comparing the two, we infer that the $2.0-\mathrm{eV}$ feature is a long-axis plasmon mode that is most strongly excited at the top of the nanoparticle. Similarly the CL and EELS profiles of the 2.8-eV feature (Figure 1d and 1e) reveal it to be a short-axis plasmon mode most strongly excited at the bottom of the nanoparticle. 
The 2.4-eV feature is more complex, exhibiting a unique spatial distribution from the CL (Figure 1f), while the EELS profile (Figure 1g) is barely distinguishable from the EELS profile of the short-axis plasmon mode shown in Figure 1e. The similarity arises because the EELS signal at $2.4 \mathrm{eV}$ is dominated by the tail of the short-axis plasmon, indicating no distinct feature is present at $2.4 \mathrm{eV}$ in the EEL spectrum, while a distinct feature at $2.4 \mathrm{eV}$ is present in the CL spectrum.

We now invoke the complementarity of EELS and CL, excitation and decay. The two spectroscopies react oppositely in the case of thick samples. Since EELS measures excitation, thicker samples result in increased excitation, and as a result the EEL spectrum of a thick sample is dominated by bulk effects that scale with sample thickness, as opposed to surface effects whose cross-sections remains unchanged. Conversely, the CL is insensitive to bulk effects. For light in the visible range, the skin depth of silver is less than $15 \mathrm{~nm}$. Hence, any emission coming from the bulk is reabsorbed and not detected efficiently. So for all samples, regardless of thickness, the CL spectrum is dominated by surface effects.

The presence of the 2.4-eV peak in the CL, coupled with its absence in EELS, assures us that the feature is a surface effect localized above the body of the nanoparticle. Since the peak shows no components along the edges of the nanoparticle, where EELS would detect it easily, we conclude that it is an out-ofplane longitudinal plasmon mode, oscillating parallel to the electron beam

With only a CL or EELS analysis, the full plasmonic behavior of the nanoparticle would not be fully understood, and simulations based on estimations of its precise morphology would be needed in order to determine the full plasmonic response of the nanoparticle. However, with the combination of CL and STEM a determination of the LSPR response of a complex nanostructure in three dimensions can be achieved using solely experimental data [5].

References:

[1] C. E. Hoffman et al, Nano Lett. 7 (2007), p. 3612.

[2] J. Nelayah et al, Nat. Phys. 3 (2007), p. 348.

[3] E. M. Pressai et al, Nano Lett. 10 (2010), p. 2097.

[4] A. Losquin et al, Nano Lett. Online Early Access (Published online: Jan. 20, 2015).

[5] This work was funded by NSF-EPS-1004083, NSF-TN-SCORE, DOD-W911NF-11-1-0156, DODW911NF-13-1-0153, DE-FG02-09ER46554, DE-FG02-01ER45916, and the DOE Office of Science BES Materials Science and Engineering Division.

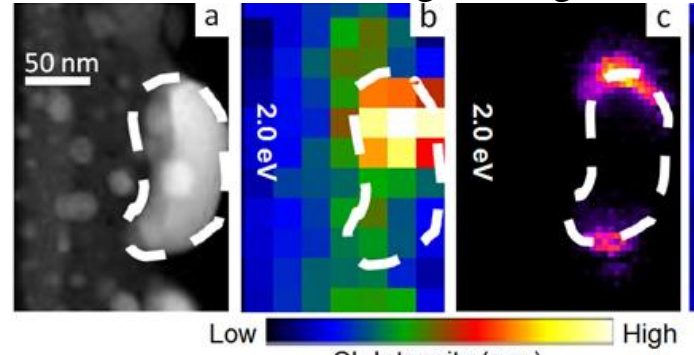

CL Intensity (a.u.)

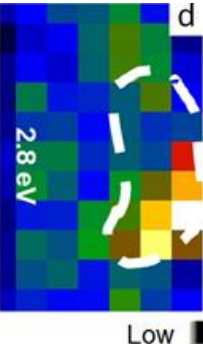

Low

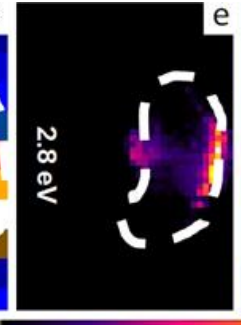

EELS Intensity (a.u.)

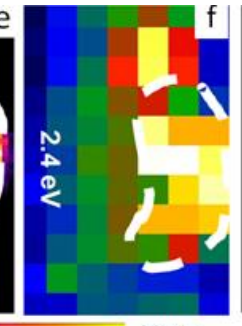

High

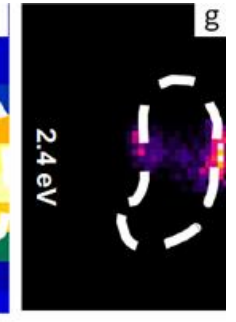

(1)

Figure 1. Plasmon mapping with complementary CL and EELS. (a) ADF image of a nanoparticle with a random shape and orientation. (b,d,f) CL and (c,e,g) EEL spectrum images of the dominant plasmon peaks. By using CL and EELS together we determine the type of mode and where it is most strongly active. (b) and (c) $2.0 \mathrm{eV}$ : Long-axis plasmon, strongest at the top of the nanoparticle. (d) and (e) $2.8 \mathrm{eV}$ : Short-axis plasmon strongest at the bottom. (f) and (g) $2.4 \mathrm{eV}$ : Out-of-plane plasmon strongest in the middle of the nanoparticle. 